\title{
Lattice model of oligonucleotide hybridization in solution. II. Specificity and cooperativity
}

\author{
J. C. Araque ${ }^{1, a)}$ and M. A. Robert ${ }^{2,3}$ \\ ${ }^{1}$ Department of Chemistry, University of Iowa, Iowa City, Iowa 52242, USA \\ ${ }^{2}$ Department of Chemical and Biomolecular Engineering, Rice University, Houston, Texas 77005, USA \\ ${ }^{3}$ Rice Quantum Institute and Richard E. Smalley Institute for Nanoscale Science and Technology, \\ Rice University, Houston, Texas 77005, USA
}

(Received 17 September 2015; accepted 26 February 2016; published online 22 March 2016)

\begin{abstract}
Because oligonucleotides are short sequences of nucleic acid bases, their association in solution with complementary strands (hybridization) is often seen to conform to a simple two-state model. However, experimental evidence suggests that, despite their short length, oligonucleotides may hybridize through multiple states involving intermediates. We investigate whether these apparently contradictory scenarios are possible by imposing different levels of sequence specificity on a lattice model of oligonucleotides in solution, which we introduced in Part I [J. C. Araque et al., J. Chem. Phys. 134, 165103 (2011)]. We find that both multiple-intermediate (weakly cooperative) and two-state (strongly cooperative) transitions are possible and that these are directly linked to the level of sequence specificity. Sequences with low specificity hybridize (base-by-base) by way of multiple stable intermediates with increasing number of paired bases. Such intermediate states are weakly cooperative because the energetic gain from adding an additional base pair is outweighed by the conformational entropy loss. Instead, sequences with high specificity hybridize through multiple metastable intermediates which easily bridge the configurational and energetic gaps between singleand double-stranded states. These metastable intermediates interconvert with minimal loss of conformational entropy leading to a strongly cooperative hybridization. The possibility of both scenarios, multiple- and two-states, is therefore encoded in the specificity of the sequence which in turn defines the level of cooperativity. (C) 2016 AIP Publishing LLC. [http://dx.doi.org/10.1063/1.4943577]
\end{abstract}

\section{INTRODUCTION}

It is generally accepted that equilibrium between singleand double-stranded oligonucleotides in aqueous solution obeys simple thermodynamic rules. In particular, if the association is assumed to be bimolecular, the progress of the hybridization as function of temperature is seen to follow the thermodynamic van't Hoff relation. ${ }^{1}$ This suggests a cooperative equilibrium model where only two states are thermodynamically accessible ${ }^{2}$ one state being the denatured (single-stranded) and the other the native duplex (doublestranded). This is also known as the all-or-none model, where the words all and none refer to the number of bases paired per duplex.

Predictive methods based on these simple principles, in particular the nearest-neighbor approach, ${ }^{3}$ have found wide acceptance for designing nucleic acid sequences. Several technological applications include genetics, ${ }^{4-6}$ drug delivery, ${ }^{7,8}$ biological and electrochemical sensors, ${ }^{9,10}$ nanostructured materials, ${ }^{11-14}$ nanomachines, ${ }^{15-18}$ and DNA-based computing. ${ }^{19-21}$ Although in most cases these predictive methods are rather reliable, they are not always consistent with experiment. ${ }^{22,23}$ Their two main shortcomings are (i) the experimentally acquired parameters and (ii) the underlying

\footnotetext{
a) Author to whom correspondence should be addressed. Electronic mail: juan-araque@uiowa.edu
}

model. The former is typically remediated by measuring thermal melting of new combinations of sequences in order to account for a larger set of sequences. ${ }^{24-27}$ For example, a set of improved nearest-neighbor parameters was obtained recently from single-molecule force-melting experiments. ${ }^{28}$ The problem with this approach is that it only provides slight increments in reliability while still propagating modeldependent approximations. ${ }^{23}$ In contrast, the underlying model requires either a more fundamental understanding or a more rigorous treatment. Indeed, the simple twostate model has been challenged by a growing body of experimental evidence favoring mechanisms with multiple intermediates. ${ }^{29-40} \mathrm{~A}$ generalized approach capable of embracing the complete combinatorial space of sequences is yet to be found.

The reason why the nearest-neighbor model and similar approaches are in general reliable is simple; a large subset of sequences hybridize through strongly cooperative two-state transitions where the multiplicity of possible intermediate states becomes statistically (or kinetically) irrelevant. ${ }^{41}$ The remaining subset, however, contains sequences prone to form intermediates because of their weak degrees of hybridization cooperativity. For instance, a recent experimental study revealed the formation of unexpected offpath intermediates for oligonucleotides sequences explicitly designed to avoid secondary structure and non-two-state hybridization. ${ }^{40}$ Another example is that of sequences 
prone to form unimolecular off-path intermediates. ${ }^{42}$ The existence of non-two-state sequences is also verified in cases where enthalpies from calorimetric (model-independent) measurements are in disagreement with those from van't Hoff (model-dependent) estimates. ${ }^{43}$ It is therefore clear that both subsets, two- and non-two-state, exist and might be related to different hybridization pathways, either thermodynamically or kinetically dominated. That behavior imitates the wellknown mechanisms of folding cooperativity in proteins. ${ }^{44}$ Most likely, DNA hybridization and protein folding share a common statistical mechanical description as well as a number of thermodynamical signatures.

In the case of single-duplex denaturation (or "melting"), a number of established theoretical ${ }^{45}$ and simulation ${ }^{46-51}$ models exist. However, the case of multiple strands in solution with all their possible hybridization pathways needs further understanding beyond that based on simplifying assumptions. ${ }^{2,52,53}$ The main problem is that severe lengthand time-scale restrictions arise from the dilute concentrations at which hybridization experiments are typically performed. Capturing a statistically reliable number of hybridization events in solution is therefore out of the reach of very detailed computational models. ${ }^{54-58}$ This is because entropic barriers associated with random collisions ${ }^{59}$ and energetic barriers associated with base pairing/unpairing ${ }^{60}$ often lead to long hybridization times in solution. ${ }^{39}$ Several computational studies of DNA melting have resorted to (umbrella sampling type) constraints that artificially hold (or bring) a pair of strands in close proximity in order to investigate transition pathways and mechanisms of single-duplex melting/hybridization. ${ }^{48,49,55,61,62}$ Although these studies capture some aspects of the problem, the imposed bias may induce a decrease in the entropy of hybridization with respect to that in solution. ${ }^{63}$ Other coarse-grained models avoid using the biasing potential by confining a pair of small DNA within finite boundaries or very small periodic boxes. ${ }^{51,63-65}$ Thermodynamic arguments are then used to extrapolate single-duplex results to insolution conditions. ${ }^{66}$ It is clear that whereas the transition entropy in these calculations can be corrected, the impact on the overall hybridization pathways/mechanisms is yet unknown.

In the present work, we aim at understanding the effect of sequence specificity on both transition cooperativity and microscopic pathways associated with hybridization in solution. We are particularly interested in describing how sequence specificity is expressed in the free-energy landscape ${ }^{67}$ either as stable or as metastable minima or as saddle points (transition states). These calculations, however, require a thorough statistical sampling of phase space and therefore beg for a model simple enough to do so. This is the reason why we resort to a lattice model of DNA oligonucleotides we recently developed. ${ }^{68}$ Indeed, in our previous work, ${ }^{68}$ we showed how this lattice model is capable of capturing fluctuations related to the effect of strand concentration on the hybridization transition (in qualitative agreement with experiment). Furthermore, our lattice model is closely analogous to that of proteins on high-coordination lattices $^{69}$ which, together with the free-energy landscape description, ${ }^{44}$ proved instrumental in elucidating the existence of several protein folding mechanisms.

Given the reduced number of degrees of freedom in our model, we demonstrate that it is possible to overcome energetically trapped states and long-diffusive time scales by our direct simulations. Despite the absence of explicit solvent effects, many aspects relevant to hybridization mechanisms in solution are captured by our model in qualitative agreement with experiment. We propose here a detailed description of how sequence specificity shapes the free-energy landscape by two distinct mechanisms (described in Sections III B and III C). Our main conclusion is that both types of hybridization pathways, either (strongly cooperative) twostate or (weakly cooperative) non-two-state, are possible depending on the level of sequence specificity. Cooperativeencoding sequences efficiently compensate energetic and entropic penalties whereas non-cooperative-encoding ones do not.

\section{MOLECULAR MODEL AND SIMULATION METHODS}

\section{A. Coarse-grained lattice model of oligonucleotides}

Single strands are considered as self- and mutually avoiding walks on a cubic lattice of coordination number $z=26 .^{70,71}$ In this representation, schematically illustrated in Fig. 1(a) of Ref. 68, successive beads of a chain are joined by a vector from the set $(0,0,1),(0,1,1)$, and $(1,1,1)$, and by equivalent vectors resulting from reflections on the cubic lattice. Nucleotide units, consisting of the sugar-phosphate backbone and any one type of nucleobase (A, T, C, or G), are coarse-grained into the monomeric units of model chains; in this way, DNA-type heterogeneity in the interactions and in the sequence distribution along the single strands are incorporated explicitly, i.e., model oligonucleotides can be homo- or hetero-oligomers according to the imposed sequence. This implies that joined segments in the lattice mapping, instead of replacing a statistical Kuhn segment, play the role of the backbone bonds that interconnect neighboring nucleotides on the actual DNA molecule.

The coarse-grained monomers in this implementation are not structureless. They contain an internal degree of freedom that accounts for the orientation of the nucleobase with respect to the backbone. This is pictorially represented by the black pins protruding from the monomers in Figs. 1(a) and 1(b) of Ref. 68. This explicit directionality, enforcing the alignment of basis vectors on the lattice, accounts for the orientation dependence of base-stacking and base-pairing interactions. Both interactions are modeled as square-well potentials with an angular component (see Figs. 2(a) and 2(b) of Ref. 68). On the lattice, this angular constraint implies that two neighboring nucleotides (within the range of $1-\sqrt{ } 3$ lattice units) can pair if they point directly towards each other. In the case of intra-strand stacking, neighboring nucleotides interact if they point in the same direction. Model details and simulation methodologies are extensively discussed in Ref. 68.

We consider model oligonucleotides with $M=8$ (8-mer) and $M=12$ (12-mer) nucleobases (considering 1 nucleobase per lattice monomer) on a three-dimensional cubic lattice with 
box length $L=30$. The number of lattice chains $N$ in each case was specified in order to have approximately the same number of monomers in both 8-mer and 12-mer systems. For 8-mer systems, the number of chains was set to $N=20$, which leads to a monomer concentration $N \times M / V=0.0059$, whereas for 12-mer systems, $N=14$ chains were considered to obtain a monomer concentration $N \times M / V=0.0062$. The substantial number of Monte Carlo steps $\left(10^{8}-10^{9}\right)$ which included enhanced sampling moves (based on configurationalbias sampling) together with feedback-optimized parallel tempering steps guaranteed proper convergence in each of the systems simulated. A detailed explanation of these aspects can be found in Ref. 68 and its associated supplementary information file.

\section{B. Free-energy landscapes from histogram reweighting}

The histogram reweighting method of Ferrenberg and Swendsen, ${ }^{72}$ as expanded by Kumar et al. ${ }^{73}$ is used to calculate population distributions and free-energy landscapes at any temperature. The two-dimensional potential of mean force $W_{T_{i}}\left(N_{\mathrm{BP}}, E\right)$ at arbitrary $T_{i}$ temperature is estimated from

$$
W_{T_{i}}\left(N_{\mathrm{BP}}, E\right)=-k_{B} T_{i} \ln \left[\frac{\mathcal{P}_{T_{i}}\left(N_{\mathrm{BP}}, E\right)}{\mathcal{P}_{T_{i}}\left(0, E_{\min }\right)}\right],
$$

where $N_{\mathrm{BP}}$ is the number of bases paired, $E$ is the total energy, and $\mathcal{P}_{T_{i}}\left(N_{\mathrm{BP}}, E\right)$ is the reweighted or composite probability distribution along the reaction coordinates calculated from

$$
\mathcal{P}_{T_{i}}\left(N_{\mathrm{BP}}, E\right)=\frac{\sum_{n=1}^{R} f_{n}\left(N_{\mathrm{BP}}, E\right) \exp [-\beta(E)]}{\sum_{m=1}^{R} K_{m} \exp \left[-\beta_{m} E-C_{m}\right]},
$$

where $K_{m}$ is the total number of observations $\left(K_{m}\right.$ $=\sum_{N_{\mathrm{BP}}, E} f_{m}\left(N_{\mathrm{BP}}, E\right)$ ) for run $i$. The constants $C_{m}$ (also known as "weights") are obtained by iteration from the relationship

$$
C_{m}=\sum_{N_{\mathrm{BP}}} \sum_{E} \mathcal{P}\left(N_{\mathrm{BP}}, E ; T_{m}\right) .
$$

Our feedback-optimized parallel tempering simulations allow for a thorough sampling of the required two-dimensional $f_{T_{i}}\left(N_{\mathrm{BP}}, E\right)$ histograms at each temperature. These histograms are then efficiently combined by using the Weighted Histogram Analysis Method (WHAM) extension of the FerrenbergSwendsen algorithm. It is verified that combining parallel tempering (feedback-optimized) with WHAM, as described by Chodera et al. ${ }^{74}$ and Rick, ${ }^{75}$ indeed improves the convergence of the estimated hybridization free-energy landscapes.

\section{RESULTS AND DISCUSSION}

To assess the effect of sequence specificity and how it may relate to hybridization cooperativity, a selected number of sequences is considered. While emphasis is on the effect of sequence complexity, in line with of Refs. 24 and 30, other parameters such as chain length and degree of complementarity are also examined as they pertain to sequence specificity. The set of four (main) sequences discussed in detail include two 8-mers and two 12-mers having 50\% and $100 \%$ content of AT base-pairs, respectively. In the interest of clarity, we shall refer to these sequences throughout the rest of the text in the following manner: CG-core 8-mer, $\left(5^{\prime} \text {-ATCGCGAT- } 3^{\prime}\right)_{2}$; AT-core 8-mer, (5'-CGATATCG- $\left.{ }^{\prime}\right)_{2}$; oligoA-oligoT 12-mer, $\left(5^{\prime}-\mathrm{A}_{12}-3^{\prime}\right) \cdot\left(3^{\prime}-\mathrm{T}_{12}-5^{\prime}\right)$; and oligoAT 12-mer, $\left(5^{\prime}-\mathrm{A}_{6} \mathrm{~T}_{6}-3^{\prime}\right)_{2}$. The structure and length of other sequences are explicitly given when they are mentioned.

\section{A. Population distributions of denatured, intermediate, and duplex species}

These populations correspond to individual thermodynamic states having the same number of paired bases $N_{\mathrm{BP}}$ in all possible microscopic configurations. Approximate distributions can be calculated theoretically from experimental data of oligonucleotide systems. ${ }^{52,76}$ In such an approach, the simplifying assumptions of the zipper model are required. In the present work, however, histograms of denatured and base-paired species populations are obtained by sampling equilibrium configurations from the explicit in-solution simulations. The distribution of states so obtained considers paired bases from distinct strands including both native and non-native contacts. Whereas native contacts correspond to A-T and G-C pairings aligned as in the fully paired duplex, non-native contacts correspond to misaligned A-T and C-G pairings. Self-association contacts formed by complementary bases on the same strand are excluded from the analysis, but these are rare and rather transient given the lattice constraints and the chain lengths considered. The resulting distributions are shown in Figs. 1(a)-1(d).

Fig. 1(a) shows that for the CG-core 8-mer sequence, a broad and significantly populated ensemble of intermediates (I) appears at the melting temperature $T_{m}$ (middle panel). These intermediates persist even at temperatures lower than $T_{m}$, where the native duplex $(\mathrm{N})$ is expected to dominate. Above $T_{m}$, the native duplex is largely suppressed and basepaired species are dominated by (nucleation) intermediates having $N_{\mathrm{BP}}=3-5$ paired bases. The broadly distributed intermediate state only shifts to higher values of $N_{\mathrm{BP}}$ in a (zipping) base-by-base manner as the temperature decreases. Thus, whereas nucleation is energetically favored at the CG-core, it is the entropically favored fraying ends (at the AT-rich terminals) which lead to the prevalent suppression of the native duplex state. Similar population distributions of partially bonded structures were observed in the nuclear magnetic resonance (NMR) spectra of (AAGCUU) $2 .{ }^{29}$ This is a ribosyl 6-mer which, having the same sequence structure of our CG-core 8-mer, also displays a weakly cooperative and multi-state transition.

The AT-core 8-mer sequence, although having the same nucleobase composition as the CG-core 8-mer, displays instead a well defined and strongly cooperative two-state transition. As shown in Fig. 1(b), the specificity of the ATcore 8-mer sequence leads to a population of species largely partitioned into denatured (D) and native (N) states at all temperatures. In other words, single-stranded $\left(N_{\mathrm{BP}}=0\right)$ and fully paired duplexes $\left(N_{\mathrm{BP}}=8\right)$ coexist with a negligible fraction of all other partially bound chains, except for a 

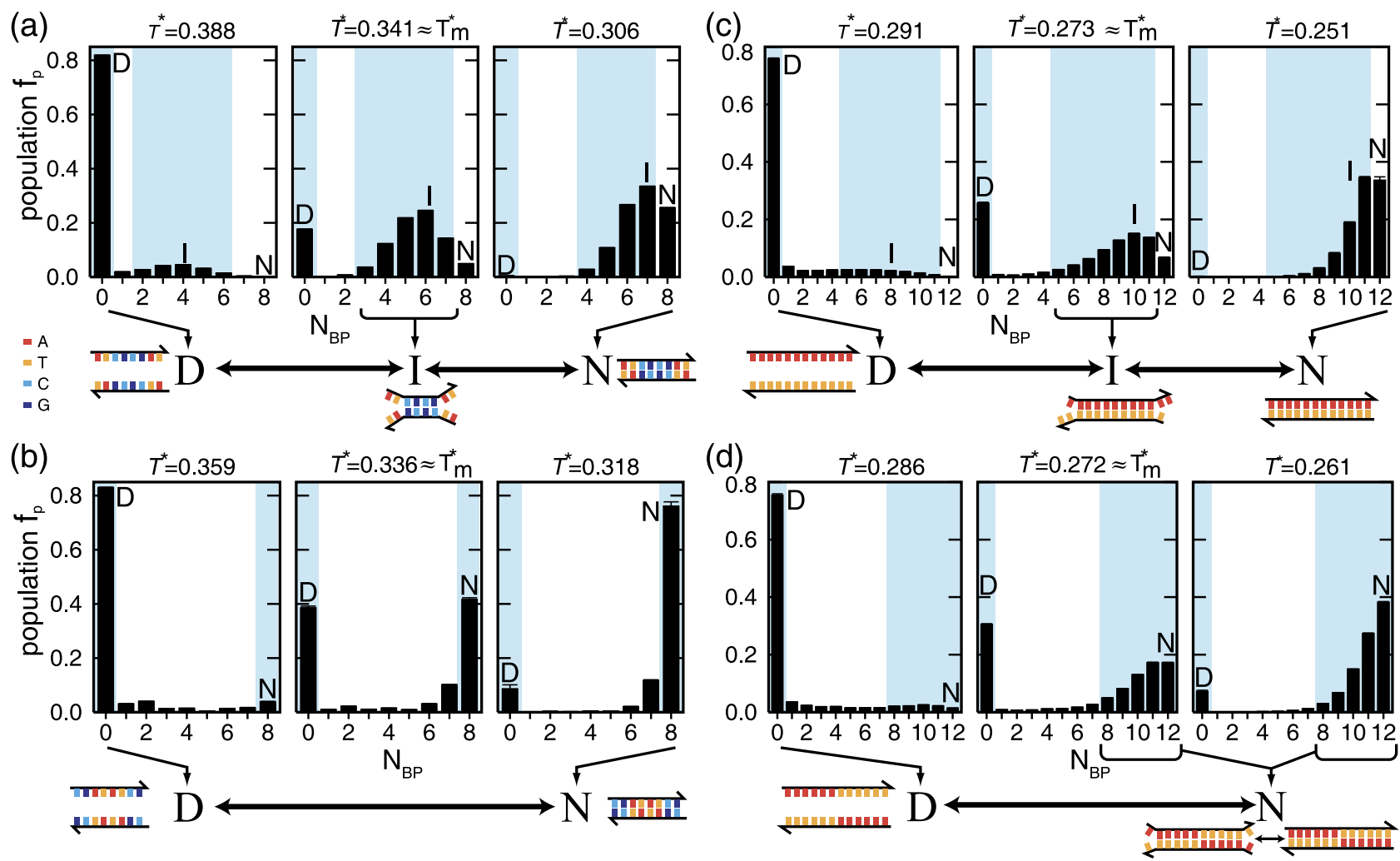

FIG. 1. Population analysis of model oligonucleotides hybridization. Histograms of population distributions and base-pairing species for the following: (a) CG-core 8-mer, (b) AT-core 8-mer, (c) oligoA-oligoT 12-mer, and (d) oligoAT 12-mer sequences. The location of denatured (D) with $N_{\mathrm{BP}}=0$, native duplex (N) with $N_{\mathrm{BP}}=8$ or 12 , and intermediate (I) states are indicated on each plot with their respective schematic configurations.

relatively small population of fraying ends. Both features agree, at least qualitatively, with those from a number of NMR experiments on sequences with similar AT-core/CG-terminal structure. ${ }^{77-79}$ The mechanism for this behavior, however, is not evident from the probability distribution itself and shall be clarified in Sec. III B.

Representative snapshots of both scenarios, CG-core (on the left) and AT-core (on the right) 8-mers, at the corresponding $T_{m}$, are shown in Figs. 2(a)-2(d). A diverse population of fully, partially, and un-paired strands can be seen in the case of the CG-core 8-mer sequence (Figs. 2(a) and 2(c)). Nucleation at the CG-core and fraying ends can be clearly distinguished within the population of partially paired duplexes of the CG-core 8-mer. In contrast, in the case of the AT-core 8-mer (Figs. 2(b) and 2(d)), a well-defined all-or-none coexistence between fully and un-paired duplexes stands out.

Two roughly equivalent hybridization scenarios are exhibited for our longer 12-mer oligonucleotides. That of the oligoA-oligoT 12-mer, shown in Fig. 1(c), is largely dominated by intermediate (I) species. One readily notices the similarity between the population distributions in Fig. 1(c) (oligoA-oligoT 12-mer) and Fig. 1(a) (CG-core 8-mer). As remarked before, nucleation at the CG-core and fraying ATterminal ends is at the origin of the intermediate-dominated transition in the case of the CG-core 8-mer. In contrast, in the case of the oligoA-oligoT 12-mer it is the low specificity of the repetitive sequence which gives rise to the large population of stable intermediates. This is because such large and repetitive sequence lacks a well defined nucleation core and is thus prone to form a large number of distinct staggered-duplexes. The presence of fraying ends is in this case enhanced by the staggering itself where one A-terminal and one T-terminal are left with no neighboring bases to pair with. Calorimetric analysis has indeed shown that the $\mathrm{d}\left(\mathrm{A}_{10}\right) \cdot \mathrm{d}\left(\mathrm{T}_{10}\right)$ 10-mer analog is non-two-state and undergoes a multi-state and broad transition. ${ }^{80}$ Significant deviations from the all-or-none model have also been observed for 18-mers duplexes of adenylic and uridylic acids $\mathrm{A}(\mathrm{pA})_{17} \cdot \mathrm{U}(\mathrm{pU})_{17}{ }^{76}$

Contrary to our expectations, because of its partially repetitive structure, the population distribution of the oligoAT 12-mer sequence shown in Fig. 1(d) can be regarded as having a (weak) two-state behavior. The distribution clearly differs from that of the AT-core 8-mer duplex but is nevertheless bimodal in D and (mostly) $\mathrm{N}$. At the melting temperature $T_{m}$, peaks are enhanced at the denatured (D) state and towards an ill-defined (close to native) stable state. Below $T_{m}$, the latter is finally dominated by the native species $\left(N_{\mathrm{BP}}=12\right)$. Overall, intermediates are neither entirely suppressed nor clearly dominating over the native state. This is also shown schematically in Fig. 1(d) where it is clear that having AT/TA symmetry in the middle of the sequence minimizes the possibility of staggering and associated intermediates states. In other words, it is as if duplexes missing one to three (likely terminal) base pairs $\left(N_{\mathrm{BP}}=9-11\right)$ were part of a fast relaxation process and not of an elementary (fully stable) step.

Most likely the existence of such a process may help to elucidate conflicting experimental results involving the same type of sequences. ${ }^{81-84}$ In the experiments of Refs. 81-84, 

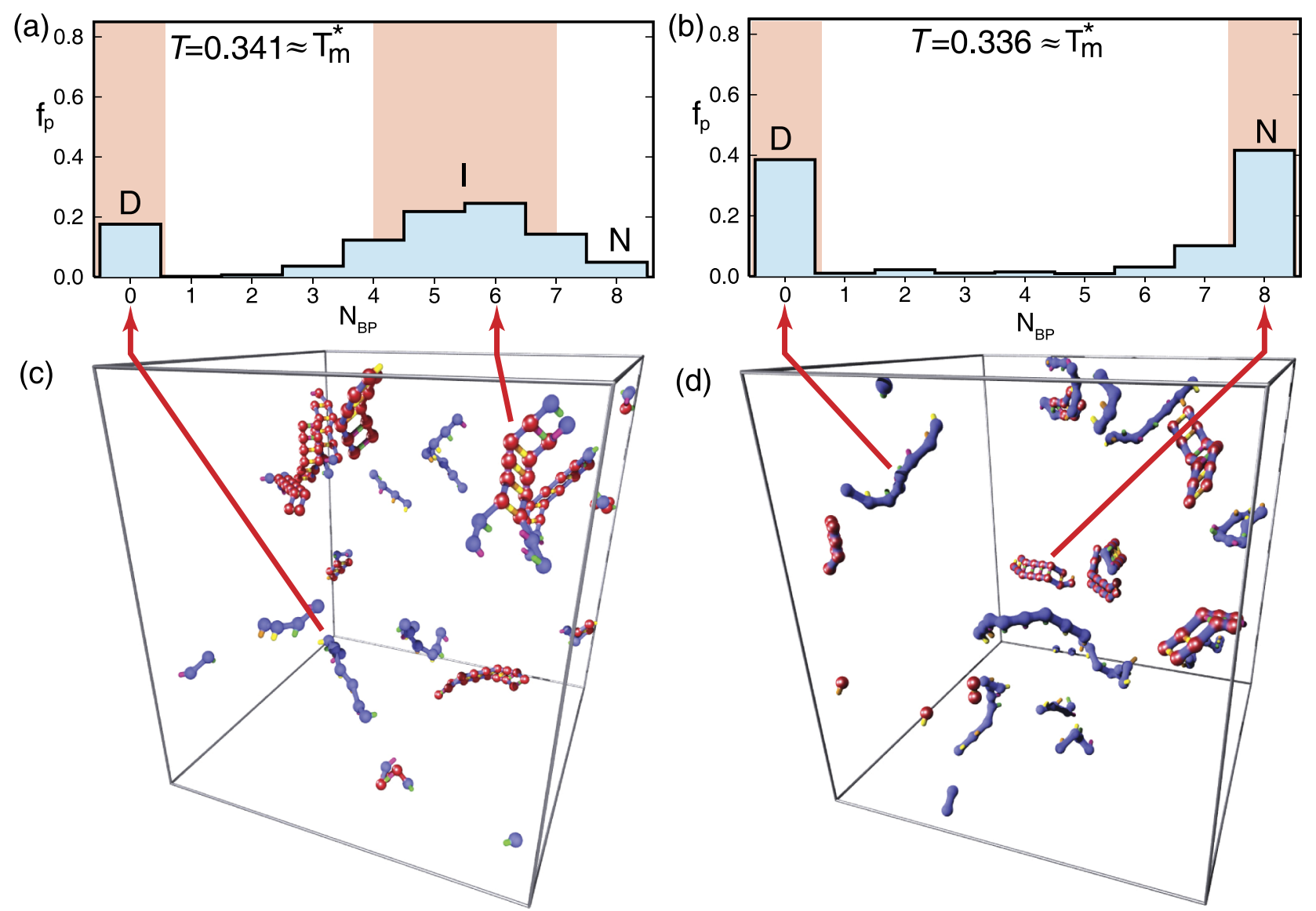

FIG. 2. Simulation snapshots of two-state and non-two-state scenarios at the transition temperature $T_{m}^{*}$. Histograms (top) and snapshots (bottom) for the following: (a) and (c) CG-core 8-mer; (b) and (d) AT-core 8-mer sequences. The location of denatured (D) with $N_{\mathrm{BP}}=0$, native duplex $(\mathrm{N})$ with $N_{\mathrm{BP}}=8$ or 12 , and intermediate (I) states are indicated on each plot. Red and blue monomers in the snapshots represent paired and un-paired bases, respectively. Color-coded pins represent nucleobase identity and directionality $(\mathrm{A}=$ green, $\mathrm{T}=$ magenta, $\mathrm{C}=$ orange, $\mathrm{G}=$ yellow $)$.

two-state conformity for a specific sequence was examined in two complementary ways: (i) by calculating the fraction $(\lambda)$ of base pairs forming cooperatively, as obtained from the ratio of van't Hoff and calorimetric enthalpies and (ii) by calculating the fraction $\left(F_{I, \max }\right)$ of intermediates from the statistical deconvolution of differential scanning calorimetry curves. For oligoribonucleotides of the type $\left(\mathrm{A}_{n} \mathrm{U}_{n}\right)_{2},{ }^{81,82}$ with $n=5$ or 7 , $\lambda$ was found to be larger than 1 (implying no intermediates), while $F_{I, \max }$ was determined to be between $20 \%$ and $25 \%$ (implying intermediates). Other studies regarding the $\left(\mathrm{A}_{7} \mathrm{U}_{7}\right)_{2}$ sequence found $\lambda$ to be less than 1 and a single intermediate state having roughly $10(\sim 70 \%)$ bases paired. ${ }^{83}$ Based on our results, we conjecture this to be related to the broad (mostly native) state shown in middle and right panels of Fig. 1(d). Further experimental studies have revealed this (weak) two-state behavior to be quite characteristic for this type of symmetric oligoribonucleotides ${ }^{84}$ This is in contrast to the case of poly $(\mathrm{A}) \cdot \operatorname{poly}(\mathrm{U})$ (oligoA-oligo $\mathrm{T}$ in our case) sequences which are dominated by stronger intermediates caused by staggering. ${ }^{85}$

\section{B. Effect of specificity on the hybridization free-energy landscapes}

Starting from the population of equilibrium microstates these landscapes are mapped onto the reduced energy $E^{*}$ and
$N_{\mathrm{BP}}$ reaction coordinates, as described in detail in Section II B. The resulting landscapes, calculated at $T_{m}$ for the same four sequences presented in Sec. III A, are shown in Fig. 3(c) and are presented alongside their corresponding $\varphi_{B}$ and $C_{v} / k_{B}$ curves in Figs. 3(a) and 3(b). In the interest of clarity, the thermodynamics features of $\varphi_{B}$ and $C_{v} / k_{B}$ will be analyzed from the perspective of the free-energy landscapes, rather than vice versa. This is because both $\varphi_{B}$ and $C_{v} / k_{B}$ are ensembleaveraged properties that result from the superposition of several underlying molecular processes. This is also the reason why $N_{\mathrm{BP}}$ is a more appropriate reaction coordinate to describe the free-energy landscapes than the natural order parameter $\varphi_{B}$ (the ensemble average of the total fraction of bases paired).

\section{AT-core 8-mer}

We first consider the case of the AT-core 8-mer sequence which was found to be fully two-state from the population analysis in Sec. III A. The topology of its hybridization landscape, shown in Fig. 3(c)(i), is noteworthy in that the transition barrier is rough and broad. Small differences between both global minima $\left(N_{\mathrm{BP}}=0\right.$ and $\left.N_{\mathrm{BP}}=8\right)$ are seen because the temperature selected is only approximately $T_{m}$. Moreover, the lowest free-energy pathway, on the top of this wide barrier, crosses two metastable intermediates 

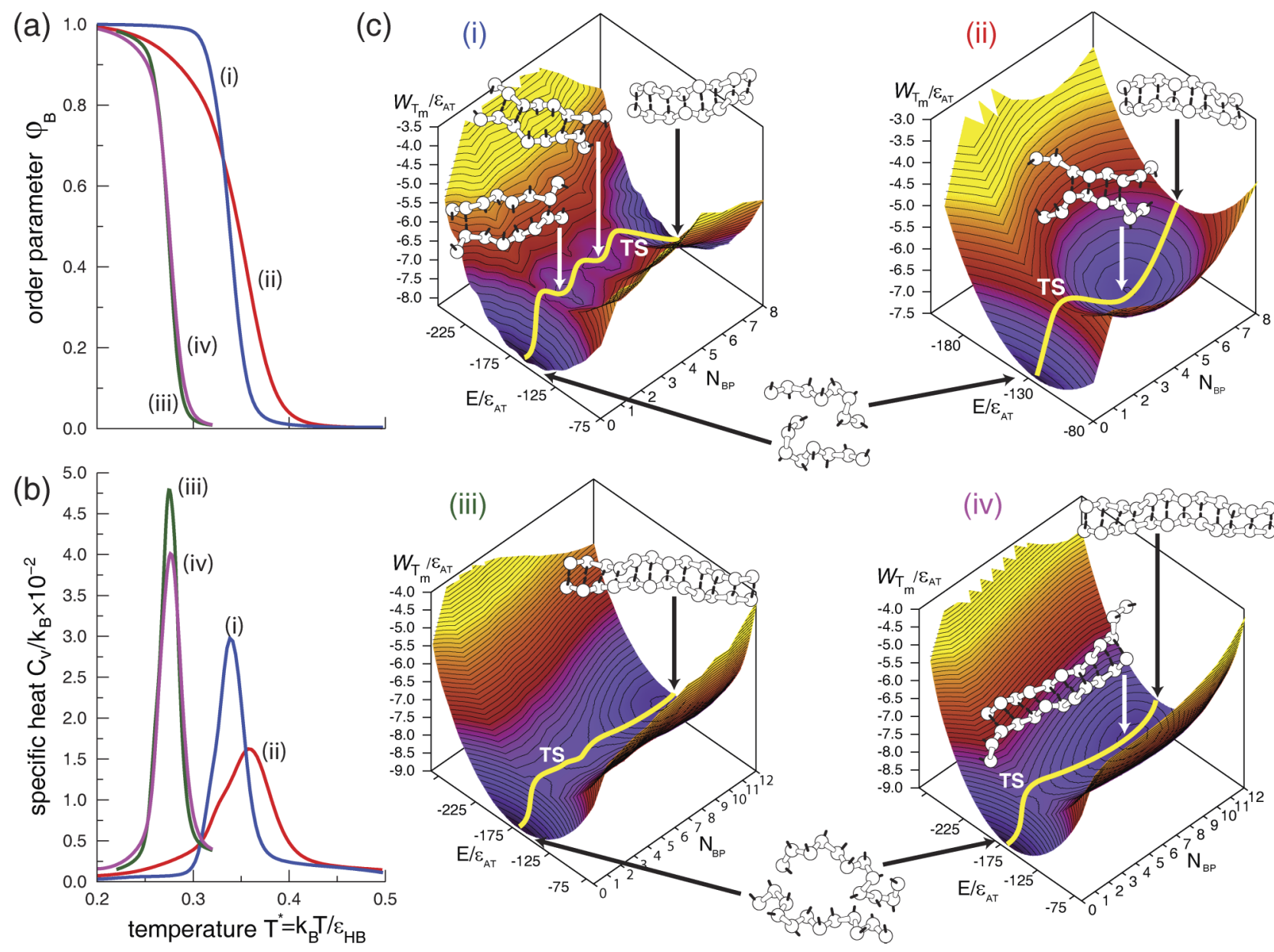

FIG. 3. Characterization of hybridization free-energy landscapes. (a) Comparison of order parameter $\varphi_{B}$ and (b) heat capacity $C_{v} / k_{B}$ curves as function of reduced temperature $T^{*}$ for: (i) AT-core 8-mer, (ii) CG-core 8-mer, (iii) oligoAT 12-mer, and (iv) oligoA-oligoT 12-mer. (c) Corresponding hybridization free-energy landscapes $W_{T m} / \varepsilon_{A T}\left(\varepsilon_{A T}\right.$ is the AT pairing energy) as functions of the reduced energy $E^{*}=E / \varepsilon_{A T}$ and number of paired bases $N_{\mathrm{BP}}$. Free-energy surfaces are calculated at the corresponding melting temperatures $T_{m}$. Representative conformations of denatured and duplex species in local and global minima are shown along the lowest free-energy pathway (yellow lines), and transition states are denoted TS. The denatured $\left(N_{\mathrm{BP}}=0\right)$ and native duplex $\left(N_{\mathrm{BP}}=8\right)$ states are indicated by black arrows and the intermediates by white arrows.

separated by a small central barrier. These local minima are bounded to the right by the main transition state TS $\left(N_{\mathrm{BP}}=5\right)$ and to the left by the nucleation step $\left(N_{\mathrm{BP}}=1\right)$. Representative configurations of the statistically dominant duplex pairings at these stages are pictured along the pathway. These, in turn, are deduced from the hybridization profiles and contact maps of base-pairing events shown in Fig. 4 (left panel of parts (a) and (b)). The metastability of these intermediates is, therefore, a consequence of the internal loop (or bubble) formed after the energetically favorable CG-pairs flanking the AT-core have nucleated (shown as a minimum at $N_{\text {BP }}=2$ ).

A helix propagation mechanism is thus suggested where both AT-pairs, directly adjacent to the flanking CG-pairs, bind first (minimum at $N_{\mathrm{BP}}=4$ ). This second local minima then promotes (by base-stacking) the formation of one additional AT-pair within the remaining unstable bubble (which is the main TS). The native duplex is then rapidly formed after both terminal CG-pairs bind. During this process the statistical ensemble of species along the barrier nucleates and grows (through small conformational rearrangements) by taking advantage of the entropy-energy compensation effect. Thus, the overall behavior just described bears a close reminiscence to the "broad barrier" $\$ 6,87$ model. In the case of proteins folding with high cooperativity (similar to our AT-core 8-mer), this mechanism accounts for both fast tunneling between unfolded and native states, and TS shifting over a broad barrier.

It is reasonable to ask whether the sequence of stages just described in the case of the AT-core 8-mer is plausible. The answer is positive when considered in conjunction with the sequence of hybridization steps derived from NMR experiments in Refs. 77-79 and 88. Indeed, these experiments show that the chemical shift and line-width transitions of resonances, along AT-core sequences, follow the same pattern as that derived from our analysis. The closest resemblance is with that of the d(GGAATTCC $)_{2}$ 8-mer sequence, ${ }^{77}$ where the three resonances corresponding to the AT-core and flanking CG-pairs were found to broaden drastically only above the melting temperature. Instead, the resonance of the terminal CG-pairs disappears much before reaching the melting transition. This could be interpreted in Figs. 3(a) and 3(b) to be the cause of the sharp transition and abrupt bends on the low- and high-temperature baselines. Longer ${ }^{79}$ 
(a)

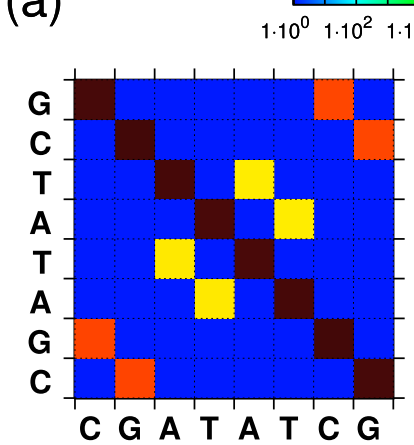

(b)

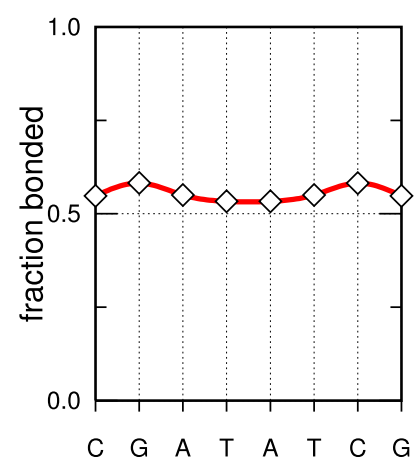

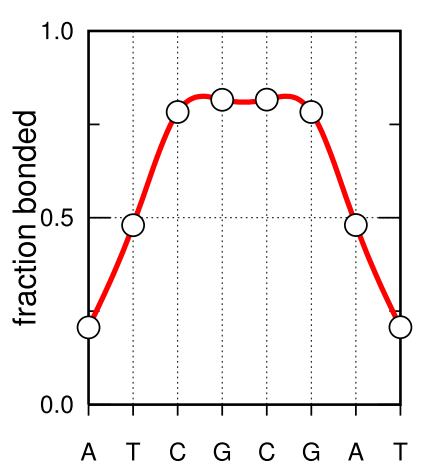

FIG. 4. Base-pairing analysis of hybridization of 8-mer sequences. (a) Contact maps of base-pairing events between all possible pairs of bases at $T_{m}$. In these maps, the bases (A, T, C, and G) along columns and rows correspond to the two complementary sequences. Thus, contacts along the main diagonal (from top-left to bottom-right) represent a perfect match, whereas off-diagonal ones are non-native contacts. (b) Base-pairing profile along the sequence at the melting transition $T_{m}$. Hybridization profiles are grouped vertically with corresponding contact maps. The logarithmic color-scale of the contact maps corresponds to the total count of contacts during the simulation.

and shorter ${ }^{78,88}$ oligonucleotides, with similar sequences, were seen to preserve both the two-state character and the barriercrossing mechanism. One can argue that, at least for the present model, the barrier broadens (or narrows) to accommodate for more (or less) transient states, while the degree of landscape roughness would also vary accordingly.

Fig. 5 shows that to be the case; both effects are seen to take place on the landscape obtained for a longer AT-core $\left(5^{\prime} \text {-CGCATATATGCG-3' }\right)_{2}$ 12-mer sequence. One observes in Fig. 5 the presence of three metastable states with deeper minima and distributed along a broader barrier than that seen for the AT-core 8-mer. Interestingly, the type of on-pathway intermediates is different for the 12-mer in that an inner AT bubble (inner loop) is not the preferred pathway, as in the case of the 8-mer (Fig. 3(c)(i)). Instead, because of the longer AT-core region, the core bases bind quickly after nucleation at one of the CG-terminals. Furthermore, the width of the barrier predicted in Fig. 5 is consistent with the size of the cooperative unit observed experimentally for an equivalent 12 -mer sequence. In that case, $\lambda$ was determined to be $\sim 0.72$ or $9 \pm 1$ bases. ${ }^{79}$ Therefore, within the context of highly cooperative two-state sequences, one may suggest that $\lambda$ accounts for the extension of the barrier rather than for the presence of stable intermediates.

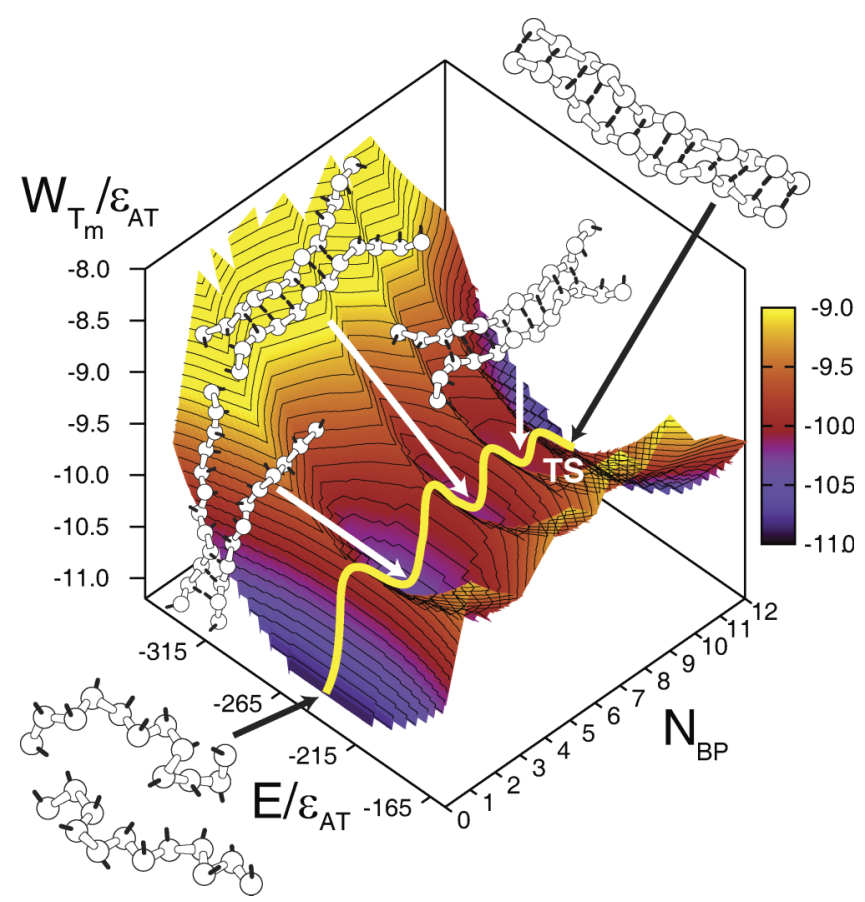

FIG. 5. Hybridization free-energy landscape $W_{T m} / \varepsilon_{A T}\left(\varepsilon_{A T}\right.$ is the AT pairing energy) as function of the reduced energy $E^{*}=E / \varepsilon_{A T}$ and number of paired bases $N_{\mathrm{BP}}$ for the (5'-CGCATATATGCG-3' $)_{2}$ 12-mer sequence. The free-energy surface is calculated at the corresponding melting temperatures $T_{m}$. Representative conformations of denatured, intermediates, and nativeduplex species are shown alongside the lowest free-energy pathway (yellow lines), and TS denotes the transition state. The denatured $\left(N_{\mathrm{BP}}=0\right)$ and native duplex $\left(N_{\mathrm{BP}}=8\right)$ states are indicated by black arrows, while the intermediates by white arrows.

\section{CG-core 8-mer}

We now turn to the analysis of the landscape topology in the case of the CG-core 8-mer (non-two-state) sequence. Fig. 3(c)(ii) shows that, in contrast to the case of the ATcore 8-mer and in accord with its lower cooperativity, a smooth double well appears, indicating a pseudo-two-state equilibrium. The denatured state (to the left) having a lower free-energy minimum than the broad (partially hybridized) intermediate state (to the right) confirms such behavior. Unequal well depths are therefore seen in this and similar cases because of this reason. It is also worth noting that the term "intermediate" in this context refers to a state that in a base-by-base manner leads to the fully hybridized state. The single barrier at the nucleation step is narrow and its freeenergy height, relative to the denatured state, is larger than that of the AT-core 8-mer sequence. Moreover, the CG-core barrier is the largest among all four sequences compared in Fig. 6. For CG-core 8-mers this can be interpreted as the result of the large entropic penalty for nucleating preferentially at the (energetically favored) CG-core. Once the CG-core has nucleated in register, the duplex conformation rapidly relaxes to the basin corresponding to the broad intermediate state.

The statistically representative conformation pictured in Fig. 3(c)(ii) was derived from the analysis of Fig. 4. Such duplex conformation is energetically stable at the core, but has a large conformational entropy originating from the fraying ends. Both effects clearly disfavor the truly native state. 


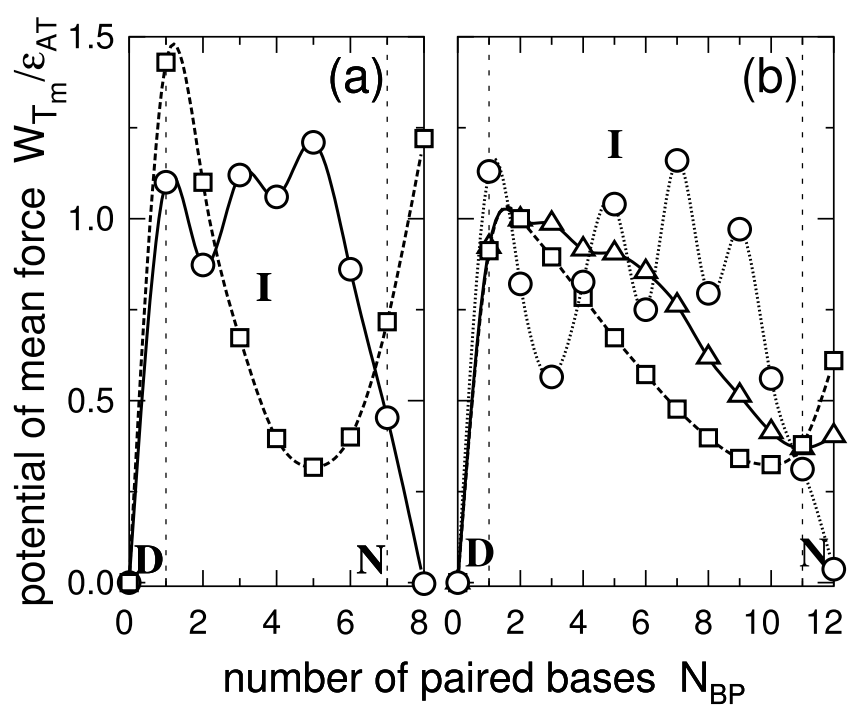

FIG. 6. Comparison of one-dimensional projections of potential of mean force as function of $N_{\mathrm{BP}}$. (a) 8-mer sequences (open circles for the AT-core, and open squares for the CG-core); (b) 12-mer sequences (open circles for the AT-core, open squares for the oligoA-oligoT, and open triangles is oligoAT). Denatured, intermediates, and native regions are denoted by D, I, and $\mathrm{N}$, respectively. Lines are guides to the eye, drawn using cubic-splines interpolation.

This is also observed in the $C_{v} / k_{B}$ curve (Fig. 3(b)), in the form of a shoulder towards the left of the main peak. Thus, the transition broadens and the characteristic $C_{v} / k_{B}$ peak decreases, whereas $\varphi_{B}$ (Fig. 3(a)) flattens out towards the low-temperature baseline. One would therefore expect the mechanism of helix propagation in this case to be kinetically controlled with base-pairing progressing gradually (base-bybase) towards the fraying ends. This is in close agreement with the mechanism proposed for the CG-core (ATCGAT) $)_{2}$ sequence from the NMR analysis of temperature- and $\mathrm{pH}-$ dependent chemical shifts and line widths. ${ }^{89}$ More recent fluorescence resonance energy transfer experiments on ATterminal-rich sequences are also in favor of the sequential (multistate) hybridization mechanism mediated by a third long-lived (intermediate) state. ${ }^{38,39}$

\section{OligoA-oligot 12-mer}

What is different in the landscape of the oligoA-oligoT 12-mer, when compared to CG-core 8-mer, is that the slope towards the stable intermediate state is less steep. This can be seen both in Fig. 3(c)(iv) and in Fig. 6. In the 12-mer case, the free-energy barrier of the critical nucleus is lower than that of the CG-core 8-mer and is not located at $N_{\mathrm{BP}}=1$, but at $N_{\mathrm{BP}}=2$ instead. This is most likely due to the low entropic penalty from nucleating with unconstrained staggering (pairing of duplexes not in register) and also because of the increased length. In addition to the entropically favorable possibility of nucleating at any position, misaligned duplexes also have the possibility of slowly realigning with modest energetic and entropic penalties by base-pair sliding. The more gradual downhill slope towards the pseudo-equilibrium intermediate in the case of the oligoA-oligoT 12-mer is likely influenced by this staggering mechanism. In support of the staggering argument, we notice that model calculations ${ }^{90}$ require the inclusion of a sliding reaction term in order to be reconciled with experimental relaxation data on homo-oligonucleotides.

\section{OligoAT 12-mer}

When comparing the nucleation barriers shown in Fig. 6 for both 100\% AT 12-mer sequences, one notices that whereas they have the same value and location, the one for the oligoAT is broader. This is a clear indication that helix propagation proceeds with larger cooperativity in that case. This is because on this slightly steep and uneven part of the landscape, further base-pairing is promoted (by hybridization with minimal penalty) rather than slowed down (by quickly proceeding downhill after nucleation). One can also see this effect in Figs. 3(c)(iii) and 3(c)(iv) by comparing both yellow pathways. We argue that the broad barrier in the case of the oligoAT 12-mer is due to the presence of the symmetry element in the middle of the sequence. This element favorably limits the amount of staggering while also inhibiting basepair sliding. The contact maps shown in Fig. 7 support this reasoning. This cooperativity-enhanced behavior, in relation to that of the oligoA-oligoT, is similarly seen in Figs. 3(a) and 3 (b), mainly in the relative steepness of the order parameter $\varphi_{B}$ at midpoint and baselines, and in the relative heights and widths of the $C_{v} / k_{B}$ peaks.

Yet another way to appreciate this behavior is by considering what happens towards the end of the oligoAT
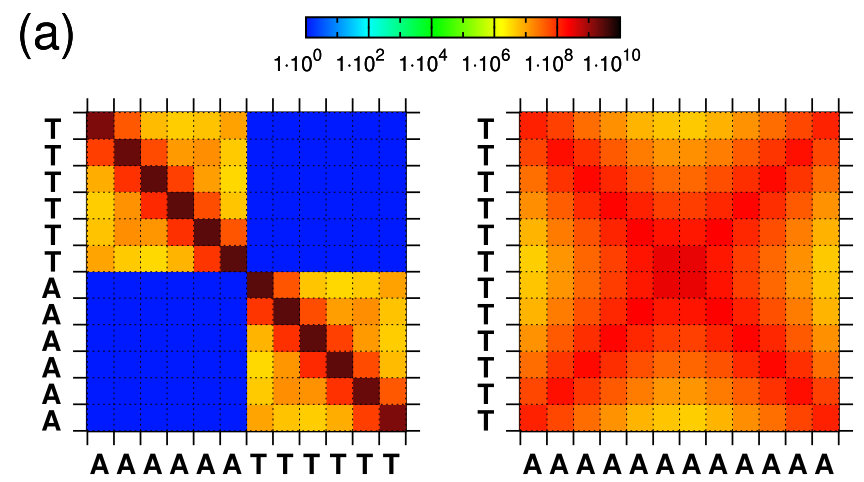

(b)
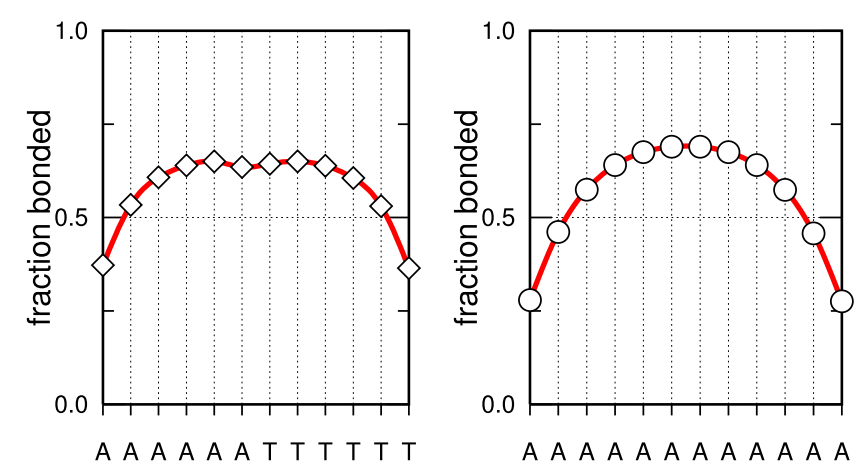

FIG. 7. Hybridization base-pairing analysis of 12-mer sequences. (a) Contact maps of base-pairing events between all possible pairs of bases at $T_{m}$; (b) base-pairing profile along the sequence at the melting transition $T_{m}$. Hybridization profiles are grouped vertically with corresponding contact maps. The logarithmic color-scale of the contact maps corresponds to the total count of contacts during the simulation. 
12-mer pathway on top of the broad barrier. In this region of the landscape, a minimum of 6 base-pairs in perfect register must have formed cooperatively, otherwise further helix propagation towards the native state would not be possible. Indeed, base-pairing is seen to propagate beyond the end of the broad barrier, albeit with lower cooperativity. The behavior of our model is consistent with that of temperature-jump experiments performed on the $\left(\mathrm{A}_{7} \mathrm{U}_{7}\right)_{2}$ sequence. ${ }^{83}$ In these experiments, one slow (end-fraying together with high-order intermediates) and one very fast (cooperative unit) relaxation signals were observed.

\section{Effect of base-pair mismatches on hybridization free-energy landscapes}

In a recent article, ${ }^{68}$ we addressed the question of whether our lattice model is capable of capturing the effect of base-pair mismatches on the duplex thermal stability. Our aim was to impose on our model a more stringent level of specificity than that of perfectly matched sequences. In other words, is it possible for a lattice model with severe dimensionality reduction to capture not only the effect of mismatches but also that of their location along the sequence? Fig. 11 in Ref. 68 shows that to be the case. Our lattice model predicted the thermal stability order of three different mismatches, relative to their (parent) perfect-matched sequence, in agreement with that predicted with the thermodynamic model of Dimitrov and Zuker. ${ }^{53}$ It is therefore enticing to delve into the study of the free-energy landscapes of mismatched sequences and to compare their topologies with that of their perfect-matched parent. Is it possible that mismatched sequences display certain degree of hybridization cooperativity in the form of broad barriers? Is their cooperativity regulated by intermediate species or by the location of the mismatched bases?

In Section II B of Ref. 68, we briefly considered the problem of cooperativity for the case of three mismatched sequences. In the present work, we analyze a pair of these sequences, derivatives of the CG-core 8-mer. Both sequences have two internal mismatches where " $C$ " nucleobases are replaced by "A" ones. In one case, the internal mismatches are located on the two middle bases of the sequence (Fig. 8(a)). In the other case, the internal mismatches are located on the two
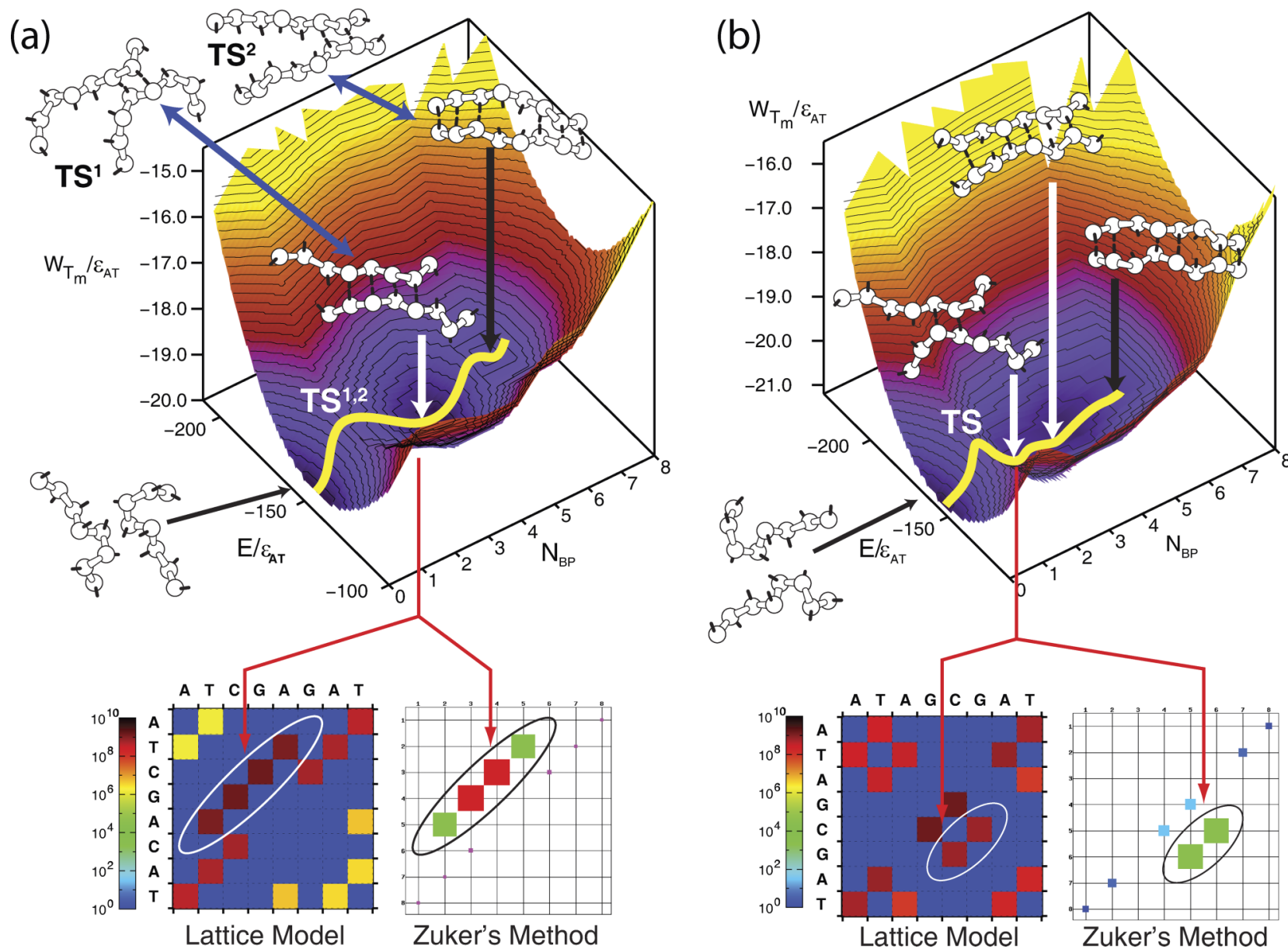

FIG. 8. Comparison of hybridization free-energy landscapes (top) and contact maps (bottom) for sequences with internal mismatches: (a) Central mismatch( $5^{\prime}$ ATCGAGAT-3' $)_{2}$ 8-mer sequence, and (b) flanking mismatch $\left(5^{\prime} \text {-ATAGCGAT-3' }\right)_{2} 8$-mer sequence. (top) Hybridization free-energy landscapes $W_{T} m / \varepsilon_{A T}\left(\varepsilon_{A T}\right.$ is the AT pairing energy) as functions of the reduced energy $E^{*}=E / \varepsilon_{A T}$ and number of paired bases $N_{\mathrm{BP}}$. Free-energy surfaces are calculated at approximately $T_{m}$ (melting temperatures). Representative conformations of denatured, intermediate, and duplex species at local and global minima are shown along the lowest free-energy pathway (yellow lines). Transition states are denoted TS and are derived from the contact maps and population analysis. The denatured $\left(N_{\mathrm{BP}}=0\right)$ and native duplex $\left(N_{\mathrm{BP}}=8\right)$ states are indicated by black arrows, whereas the intermediates are indicated by white arrows. (bottom) Contact maps for each sequence are calculated with the present model (left) and with Zuker's ${ }^{91}$ DINAMelt web server (right). The logarithmic color-scale of the contact maps (left) corresponds to the total count of contacts during the simulation. 
bases flanking the two middle bases (Fig. 8(b)). In both cases, the native state corresponds to $N_{\mathrm{BP}}=6$ instead of $N_{\mathrm{BP}}=8$. In Ref. 68, only variations in the steepness of the order parameter $\varphi_{B}$ and in the shape of the $C_{v} / k_{B}$ curve were investigated to infer the relative level of cooperativity. Instead, in Fig. 8, we analyze the same relation, but using the free-energy landscape prescription.

The case of two mismatched " $\mathrm{A}$ " bases located in the middle of the CG-core is illustrated in Fig. 8(a). Out of the two dominant transition states $\left(\mathrm{TS}^{1,2}\right.$ ), both having two nucleated bases, only $\mathrm{TS}^{1}$ is capable to form an intermediate by way of small conformational rearrangements. The off-diagonal intermediate thus formed, although having none of the native contacts of the final duplex, is energetically favorable because of the four contiguous base pairs (2AT and 2CG) in staggeredtype alignment. It is also entropically favorable because of the fraying ends. Therefore, the free-energy landscape in this case is heavily influenced by this stable intermediate which is seen as a deep minimum at $N_{\mathrm{BP}}=4$ in the top panel of Fig. 8(a). It also seen as the intense signal from four upper off-diagonal elements in the contact map shown in the left bottom panel. Interestingly, the contact map derived from thermodynamics, using Zuker's ${ }^{91}$ DINAMelt program, shows that at $T_{m}$ the same off-diagonal contacts dominate the transition.

A rather complex situation arises because the stable offdiagonal intermediate is topologically unrelated to the native $N_{\mathrm{BP}}=6$ state. This means that it cannot lead to it by way of local conformational rearrangements. Instead, the native duplex needs to be formed by further nucleation of the $\mathrm{TS}^{2}$ conformation. Nevertheless, this second nucleation is only feasible at low temperature because it needs to overcome the entropic penalty of forming a mismatched bubble (internal loop) in the middle of the sequence. One can speculate that this process would also be kinetically slow because of the low cooperativity (broad transition) seen in the specific heat plot shown in Fig. 11 of Ref. 68.

Overall, in the case of the central mismatch sequence, the formation of an intermediate (staggered) duplex, which precludes the formation of the native (in-register) duplex, leads to a poorly cooperative transition. The fact that topological constraints induce an interconversion failure (dereliction) between these two states is indicative of "entropic frustration" effects, as those seen in other biomimetic and associating polymers systems. ${ }^{92,93}$ The degrading effect of such behavior for pattern recognition purposes, as described by Muthukumar, ${ }^{94}$ is produced in this case by the introduction of a single mismatched base. This example illustrates how small changes in the sequence can have big effects in the hybridization free-energy landscape of DNA oligonucleotides. In fact, these effects are extensively exploited for highresolution analysis of DNA in microarray technologies based on single nucleotide polymorphism..$^{95}$

In contrast, the landscape shown in Fig. 8(b) for the sequence with a flanking mismatch has a broad and fairly leveled basin. Thus, the ease of conformational interconversions between the two main intermediates $\left(N_{\mathrm{BP}}\right.$ $=2,4)$ and the native state $\left(N_{\mathrm{BP}}=6\right)$ is favored by the relatively small barriers. Both (metastable) intermediates would thus be part of the same cooperative unit. Indeed, in the bottom panel of Fig. 8(b), the staggered intermediate with 2 off-diagonal contacts, and the aligned one with four, are both seen to occur in the contact maps with only a slightly larger proportion than the native (flanking-mismatched) state.

The relative cooperativity level, as derived from the analysis of Fig. 8, is seen to be compatible with that described in our previous work. ${ }^{68}$ The present analysis, however, allows us to clarify why the hybridization in the case of the central-mismatch sequence exhibits lower cooperativity (lower specific heat peak) than that of the flanking-mismatch case. The presence of metastable intermediates over a leveled free-energy landscape, with no major topological constraints, allows for a larger cooperative unit in the latter case. Nevertheless, thermodynamic model $\mathrm{s}^{53,91}$ predict the opposite order in cooperativity (specific heat peaks), while the thermal stability order $\left(T_{m}\right)$ is in agreement with ours. ${ }^{68}$ Although limitations on both approaches are obvious, our explicit simulations highlight the importance of intermediate states, and the possibility of frustrated or facilitated interconversions on the hybridization free-energy landscape.

\section{CONCLUSION}

In this study, hybridization of model oligonucleotides in solution is observed to occur by way of two distinct mechanisms: either non-two-state, if hindered by stable intermediates, or two-state, if facilitated by metastable ones. Both cases are dictated by the level of sequence specificity. Differences between them are therefore not only in terms of their thermodynamic cooperativity and multiplicity of states, but also in their mechanistic hybridization pathways. We have described such mechanistic details by reconstructing the free-energy landscape topologies from explicit in-solution simulations. When contrasted with experimental evidence on similar sequences, results from our lattice model are shown to be thermodynamically and mechanistically consistent. For instance, in the case of sequences with non-two-state (weakly cooperative) hybridization, a stable duplex intermediate is seen to readily form after nucleation. Further hybridization towards the native duplex is only possible through base-by-base pairing propagation as the temperature is lowered.

However, a more complex and unexpected picture emerged for two-state (strongly cooperative) transitions. These are seen to be characterized by landscapes with broad and moderately rough free-energy barriers. In this case, large energetic and configurational gaps between denatured and native duplex states are bridged (tunneled) by conformational rearrangements of on-pathway metastable intermediates. Such a mechanism highlights the possibility of interconversion with minimal conformational entropy loss, while favoring inter-strand base-pairing and intra-strand stacking.

The broad barrier mechanism described in the present work was previously identified in the case of protein folding ${ }^{86,87}$ but to the best of our knowledge, it has not been previously linked to oligonucleotide hybridization. This is most likely because the metastable intermediates along the broad-barrier are largely inaccessible to typical DNA experimental techniques such as ultraviolet spectroscopy, cir- 
cular dichroism, nuclear magnetic resonance, and differential scanning calorimetry. It is possible, however, that such labile and poorly populated states over broad barriers are related to kinetic intermediates seen in temperature-jump experiments. ${ }^{76,88}$ The hybridization scenarios described here using our lattice model explain and correlate a number of observations from experiment. Among the open questions that emerge from the present work are: how are the features of the free-energy landscape encoded in the nucleotide sequence? Why some sequences lead to "beneficial" intermediates, which cooperatively bridge the denatured and native duplex states, while others do not. Answering these questions will prove advantageous to improving the predictive capacity of thermodynamic hybridization models.

\section{ACKNOWLEDGMENTS}

The authors are grateful to Professor A. Z. Panagiotopoulos for helpful discussions. Support at Rice University was provided by the National Science Foundation (Grant No. CTS0327487), the Welch Foundation, and by the Rice Terascale Cluster funded by NSF (Grant No. EIA-0216467). Finally, the authors thank the referees for insightful and constructive criticisms.

${ }^{1}$ C. R. Cantor and P. R. Schimmel, Biophysical Chemistry, Part III: The Behavior of Biological Macromolecules (W. H. Freeman, San Francisco, CA, 1980).

${ }^{2}$ K. J. Breslauer, Methods Enzymol. 259, 221 (1995).

${ }^{3}$ P. N. Borer, B. Dengler, and I. Tinoco, J. Mol. Biol. 86, 843 (1974).

${ }^{4}$ M. Schena, D. Shalon, R. W. Davis, and P. O. Brown, Science 270, 467 (1995).

${ }^{5}$ A. Fire, S. Xu, M. K. Montgomery, S. A. Kostas, S. E. Driver, and C. C. Mello, Nature 391, 806 (1998).

${ }^{6}$ N. L. Rosi, D. A. Giljohann, C. S. Thaxton, A. K. R. Lytton-Jean, M. S. Han, and C. A. Mirkin, Science 312, 1027 (2006).

${ }^{7}$ Y. S. Choi and J. R. Baker, Cell Cycle 4, 669 (2005).

${ }^{8}$ Y. S. Choi, T. Thomas, A. Kotlyar, M. T. Islam, and J. R. Baker, Chem. Biol. 12, 35 (2005).

${ }^{9}$ T. A. Taton, C. A. Mirkin, and R. L. Letsinger, Science 289, 1757 (2000).

${ }^{10}$ G. Liu and Y. Lin, J. Am. Chem. Soc. 129, 10394 (2007).

${ }^{11}$ M. P. Valignat, O. Theodoly, J. C. Crocker, W. B. Russel, and P. M. Chaikin, Proc. Natl. Acad. Sci. U. S. A. 102, 4225 (2005).

${ }^{12}$ M. R. Jones, R. J. Macfarlane, B. Lee, J. Zhang, K. L. Young, A. J. Senesi, and C. A. Mirkin, Nat. Mater. 9, 913 (2010).

${ }^{13}$ X. Wei, J. Nangreave, and Y. Liu, Acc. Chem. Res. 47, 1861 (2014).

${ }^{14}$ M. Saoji, D. Zhang, and P. J. Paukstelis, Biopolymers 103, 618 (2015).

${ }^{15}$ S. J. Green, D. Lubrich, and A. J. Turberfield, Biophys. J. 91, 2966 (2006)

${ }^{16}$ J. Bath and A. J. Turberfield, Nat. Nanotechnol. 2, 275 (2007).

${ }^{17}$ T. E. Tomov, R. Tsukanov, M. Liber, R. Masoud, N. Plavner, and E. Nir, J. Am. Chem. Soc. 135, 11935 (2013).

${ }^{18}$ T.-G. Cha, J. Pan, H. Chen, H. N. Robinson, X. Li, C. Mao, and J. H. Choi, J. Am. Chem. Soc. 137, 9429 (2015).

${ }^{19}$ L. Adleman, Science 266, 1021 (1994).

${ }^{20}$ E. Shapiro and Y. Benenson, Sci. Am. 294, 45 (2006).

${ }^{21}$ V. M. Cervantes-Salido, O. Jaime, C. A. Brizuela, and I. M. Martnez-Prez, Appl. Soft Comput. 13, 4594 (2013).

${ }^{22}$ A. Panjkovich and F. Melo, Bioinformatics 21, 711 (2005).

${ }^{23}$ D. Tulpan, M. Andronescu, and S. Leger, BMC Bioinf. 11, 105 (2010).

${ }^{24}$ K. J. Breslauer, R. Frank, H. Blocker, and L. A. Marky, Proc. Natl. Acad. Sci. U. S. A. 83, 3746 (1986).

${ }^{25}$ N. Sugimoto, S. Nakano, M. Yoneyama, and K. Honda, Nucleic Acids Res. 24, 4501 (1996).

${ }^{26}$ J. SantaLucia, H. T. Allawi, and P. A. Seneviratne, Biochemistry 35, 3555 (1996).

${ }^{27}$ J. SantaLucia, Proc. Natl. Acad. Sci. U. S. A. 95, 1460 (1998).

${ }^{28}$ J. M. Huguet, C. V. Bizarro, N. Forns, S. B. Smith, C. Bustamante, and F. Ritort, Proc. Natl. Acad. Sci. U. S. A. 107, 15431 (2010).

${ }^{29}$ P. N. Borer, L. S. Kan, and P. O. P. Ts'o, Biochemistry 14, 4847 (1975).
${ }^{30}$ A. Montrichok, G. Gruner, and G. Zocchi, Europhys. Lett. 62, 452 (2003).

${ }^{31}$ Y. Gao, L. K. Wolf, and R. M. Georgiadis, Nucleic Acids Res. 34, 3370 (2006).

${ }^{32}$ D. Andreatta, S. Sen, J. L. Prez-Lustres, S. A. Kovalenko, N. P. Ernsting, C. J. Murphy, R. S. Coleman, and M. A. Berg, J. Am. Chem. Soc. 128, 6885 (2006).

${ }^{33}$ M. T. Woodside, P. C. Anthony, W. M. Behnke-Parks, K. Larizadeh, D. Herschlag, and S. M. Block, Science 314, 1001 (2006).

${ }^{34}$ P. J. Mikulecky and A. L. Feig, Biochemistry 45, 604 (2006).

${ }^{35}$ H. Ma, C. Wan, A. Wu, and Z. H. Zewail, Proc. Natl. Acad. Sci. U. S. A. 104, 712 (2007).

${ }^{36}$ C. Chen, W. Wang, Z. Wang, F. Wei, and X. S. Zhao, Nucleic Acids Res. 35, 2875 (2007).

${ }^{37}$ E. Carrillo-Nava, Y. Mejia-Radillo, and H.-J. Hinz, Biochemistry 47, 13153 (2008).

${ }^{38}$ X. Chen, Y. Zhou, P. Qu, and X. S. Zhao, J. Am. Chem. Soc. 130, 16947 (2008).

${ }^{39}$ Y. Yin and X. S. Zhao, Acc. Chem. Res. 44, 1172 (2011).

${ }^{40}$ J. R. Sikora, B. Rauzan, R. Stegemann, and A. Deckert, J. Phys. Chem. B 117, 8966 (2013).

${ }^{41}$ B. Rauzan, E. McMichael, R. Cave, L. R. Sevcik, K. Ostrosky, E. Whitman, R. Stegemann, A. L. Sinclair, M. J. Serra, and A. A. Deckert, Biochemistry 52, 765 (2013).

${ }^{42}$ E. T. Zuo, F. A. Tanious, W. D. Wilson, G. Zon, G. S. Tan, and R. M. Wartell, Biochemistry 29, 4446 (1990).

${ }^{43}$ I. Rouzina and V. A. Bloomfield, Biophys. J. 77, 3242 (1999).

${ }^{44}$ J. N. Onuchic, Z. Luthey-Schulten, and P. G. Wolynes, Annu. Rev. Phys. Chem. 48, 545 (1997).

${ }^{45}$ N. Theodorakopoulos, T. Dauxois, and M. Peyrard, Phys. Rev. Lett. 85, 6 (2000).

${ }^{46}$ K. Drukker, G. Wu, and G. C. Schatz, J. Chem. Phys. 114, 579 (2001).

${ }^{47}$ T. A. Knotts, N. Rathore, D. C. Schwartz, and J. J. de Pablo, J. Chem. Phys. 126, 084901 (2007).

${ }^{48}$ E. J. Sambriski, D. C. Schwartz, and J. J. de Pablo, Proc. Natl. Acad. Sci. U. S. A. 106, 18125 (2009).

${ }^{49}$ E. J. Sambriski, V. Ortiz, and J. J. de Pablo, J. Phys.: Condens. Matter 21, 034105 (2009).

${ }^{50}$ E. J. Sambriski, D. C. Schwartz, and J. J. de Pablo, Biophys. J. 96, 1675 (2009).

${ }^{51}$ T. E. Ouldridge, A. A. Louis, and J. P. K. Doye, J. Chem. Phys. 134, 085101 (2011).

52 J. Applequist and V. Damle, J. Am. Chem. Soc. 87, 1450 (1965).

${ }^{53}$ R. A. Dimitrov and M. Zuker, Biophys. J. 87, 215 (2004).

${ }^{54}$ M. McCullagh, T. Prytkova, S. Tonzani, N. D. Winter, and G. C. Schatz, J. Phys. Chem. B 112, 10388 (2008).

${ }^{55}$ S. Kannan and M. Zacharias, Phys. Chem. Chem. Phys. 11, 10589 (2009).

${ }^{56}$ O. S. Lee, T. R. Prytkova, and G. C. Schatz, J. Phys. Chem. Lett. 1, 1781 (2010).

${ }^{57}$ J. P. K. Doye, T. E. Ouldridge, A. A. Louis, F. Romano, P. Šulc, C. Matek, B. E. K. Snodin, L. Rovigatti, J. S. Schreck, R. M. Harrison et al., Phys. Chem. Chem. Phys. 15, 20395 (2013).

${ }^{58}$ J. J. Uusitalo, H. I. Ingalfsson, P. Akhshi, D. P. Tieleman, and S. J. Marrink, J. Chem. Theory Comput. 11, 3932 (2015).

${ }^{59}$ J. G. Wetmur and N. Davidson, J. Mol. Biol. 31, 349 (1968).

${ }^{60}$ D. H. Turner, in Nucleic Acids: Structures, Properties and Functions, edited by V. Bloomfield, D. Crothers, and I. Tinoco (University Science Press, 2000), pp. 259-334.

${ }^{61}$ T. J. Schmitt and T. A. Knotts, J. Chem. Phys. 134, 205105 (2011).

${ }^{62}$ T. J. Schmitt, J. B. Rogers, and T. A. Knotts, J. Chem. Phys. 138, 035102 (2013).

${ }^{63}$ T. R. Prytkova, I. Eryazici, B. Stepp, S. B. Nguyen, and G. C. Schatz, J. Phys. Chem. B 114, 2627 (2010).

${ }^{64}$ T. E. Ouldridge, P. Sulc, F. Romano, J. P. K. Doye, and A. A. Louis, Nucleic Acids Res. 41, 8886 (2013).

${ }^{65}$ M. Maciejczyk, A. Spasic, A. Liwo, and H. A. Scheraga, J. Chem. Theory Comput. 10, 5020 (2014).

${ }^{66}$ T. E. Ouldridge, J. Chem. Phys. 137, 144105 (2012).

${ }^{67}$ J. Kim and J.-S. Shin, Biochemistry 49, 3420 (2010).

${ }^{68}$ J. C. Araque, A. Z. Panagiotopoulos, and M. A. Robert, J. Chem. Phys. 134, 165103 (2011).

${ }^{69}$ A. Kolinski and J. Skolnick, Proteins 18, 338 (1994).

${ }^{70}$ R. G. Larson, L. E. Scriven, and H. T. Davis, J. Chem. Phys. 83, 2411 (1985)

${ }^{71}$ A. Z. Panagiotopoulos, V. Wong, and M. A. Fiorano, Macromolecules 31, 912 (1998)

${ }^{72}$ A. M. Ferrenberg and R. H. Swendsen, Phys. Rev. Lett. 63, 1195 (1989). 
${ }^{73}$ S. Kumar, J. Bouzida, R. H. Swendsen, P. A. Kollman, and J. M. Rosenberg, J. Comput. Chem. 13, 1011 (1992).

${ }^{74}$ J. D. Chodera, W. C. Swope, J. W. Pitera, C. Seok, and K. A. Dill, J. Chem. Theory Comput. 3, 26 (2007).

${ }^{75}$ S. W. Rick, J. Chem. Theory Comput. 2, 939 (2006).

${ }^{76}$ D. Porschke and M. Eigen, J. Mol. Biol. 62, 361 (1971).

${ }^{77}$ D. J. Patel and L. L. Canuel, Eur. J. Biochem. 96, 267 (1979).

${ }^{78} \mathrm{~S}$. Tran-Dinh, J. M. Neumann, T. Huynh-Dinh, P. Allard, J. Y. Lallemand, and J. Igolen, Nucleic Acids Res. 10, 5319 (1982).

${ }^{79}$ D. J. Patel, S. A. Kozlowski, L. A. Marky, C. Broka, J. A. Rice, K. Itakura, and K. J. Breslauer, Biochemistry 21, 428 (1982).

${ }^{80}$ M. Tarky, M. Bolli, and C. Leumann, Helv. Chim. Acta 77, 716 (1994).

${ }^{81}$ J. Ohms and T. Ackermann, Biophys. Chem. 34, 137 (1989).

${ }^{82} \mathrm{~J}$. Ohms and T. Ackermann, Biochemistry 27, 5237 (1990).

${ }^{83}$ K. J. Breslauer, J. M. Sturtevant, and I. Tinoco, J. Mol. Biol. 99, 549 (1975).
${ }^{84}$ D. R. Hickey and D. H. Turner, Biochemistry 24, 2086 (1985).

${ }^{85}$ E. Freire and R. L. Biltonen, Biopolymers 17, 463 (1978).

${ }^{86} \mathrm{M}$. Silow and M. Oliveberg, Biochemistry 36, 7633 (1997).

${ }^{87}$ M. Oliveberg, J. Y. Tan, M. Silow, and A. R. Fersht, J. Mol. Biol. 277, 933 (1998).

${ }^{88}$ A. P. Williams, C. E. Longfellow, S. M. Freier, R. Kierzek, and D. H. Turner, Biochemistry 28, 4283 (1989).

${ }^{89}$ D. J. Patel and C. W. Hilbers, Biochemistry 14, 2651 (1975).

${ }^{90}$ D. Porschke, Biophys. Chem. 2, 83 (1974).

${ }^{91}$ N. R. Markham and M. Zuker, Nucleic Acids Res. 33, W577 (2005).

${ }^{92}$ A. J. Golumbfskie, V. S. Pande, and A. K. Chakraborty, Proc. Natl. Acad. Sci. U. S. A. 96, 11707 (1999).

${ }^{93}$ M. Muthukumar, Comput. Mater. Sci. 4, 370 (1995).

${ }^{94}$ M. Muthukumar, Proc. Natl. Acad. Sci. U. S. A. 96, 11690 (1999).

${ }^{95}$ T. LaFramboise, Nucleic Acids Res. 37, 4181 (2009). 